\title{
History of aspirin use does not affect prognosis in patients with non-cardioembolic ischemic stroke \\ ISMAIL M, FONG WC
}

Division of Neurology, Department of Medicine, Queen Elizabeth Hospital, HKSAR

\section{Introduction and Objective}

Aspirin is commonly used as a secondary prevention treatment against ischemic stroke and transient ischemic attack (TIA). However, aspirin resistance is a common problem worldwide. The presence of aspirin resistance was found to be associated with an increased risk of recurrent stroke, death from all causes and vascular events. On the other hand, whether clinical aspirin treatment failure can predict a less optimal response to aspirin treatment in patients with acute ischemic stroke remains controversial. This study would like to determine if aspirin treatment failure predicts the prognosis of patients who had acute non-cardioembolic ischemic stroke in terms of recurrent acute ischaemic stroke, any vascular events and all causes mortality.

\section{Methods}

Patient recruitment All patients who were aged forty or above and were admitted to the acute stroke unit of Queen Elizabeth Hospital for acute ischemic stroke from $1^{\text {st }}$ October 2014 to $5^{\text {th }}$ January 2015 were screened for eligibility.

Exclusion Patients who have evidence of cardioembolism according to the TOAST criteria.

Primary outcome Recurrent acute ischaemic stroke within 24 months after the index event.

Secondary outcome Any cardiovascular events occurred within 24 months after the index event; all causes mortality

Data extraction Stroke registry of Queen Elizabeth Hospital, (prospectively collected by stroke nurses from every new stroke patient). Supplementary data as well as outcome data were traced retrospectively in the electronic clinical management system.

Statistical Analysis Overall survival is defined as the time (in months) between the date of the index event and the first recurrence. Covariates that are of known clinical interest in the Kaplan-Meyer analysis will be selected for multivariate analysis using Cox's proportional hazard models. Adjusted hazard ratios and the corresponding $95 \%$ confidence interval were reported.

\section{Patient Recruitment}

Total 264 acute ischaemic stroke admissions

98 excluded due to
cardioembolic causes; 3
excluded because of age
less than 40

Large artery

110 small vessel occlusion
Baseline Characteristics

Age (Median, IQR) 68 (60-81)

Sex (Male no, \%) $103(63.2 \%)$

Hypertension (\%) $109(66.9 \%)$

Diabetes Mellitus (\%) $44(27.0 \%)$

Hyperlipidemia (\%) $60(36.8 \%)$

History of TIA or CVA (\%) 40 (24.5\%)

History of IHD 6 months or more before stroke 17 (10.4\%)

(\%)

Use of Aspirin (\%) 43 (26.4\%)

NIHSS (median, IQR) 4 (2-8)

Type of Stroke

Large artery atherosclerosis 20 (12.3\%)

Small vessel occlusion $110(67.5 \%)$

Other ischaemic stroke $33(20.2 \%)$

Any ICA stenosis (\%) $8(6.5 \%)$

\section{Primary Outcome}

At 2 years follow up, 15 patients (9.2\%) suffered from recurrent ischaemic stroke, of which 12 patients $(7.4 \%)$ happened in the first year of follow up.

Cox regression model to predict recurrent stroke

\begin{tabular}{lccc}
\multicolumn{1}{c}{ Parameter } & RR & $\mathbf{9 5 \%}$ C.I. & $\boldsymbol{p}$ \\
Age & 1.041 & $0.996-1.088$ & 0.072 \\
Aspirin use & 0.759 & $0.222-2.603$ & 0.662 \\
LACI & 3.696 & $1.154-11.835$ & 0.028 \\
NIHSS 0-3 & 2.666 & $0.888-8.004$ & 0.080
\end{tabular}

\section{Secondary Outcome}

26 patients $(\mathbf{1 6 \%})$ suffered from a cardiovascular event during the 24 months follow up period. Apart from the 15 patients who had recurrent ischaemic stroke, 2 patients suffered from transient ischemic attack, 4 from intracerebral haemorrhage, 1 from intraventricular haemorrhage and 4 from ischaemic heart disease. The all causes mortality was $\mathbf{1 3 . 5 \%}$.

\section{Cox regression model to predict mortality}

$\begin{array}{lccc}\text { Parameter } & \mathbf{R R} & \mathbf{9 5 \%} \text { C.I. } & \boldsymbol{p} \\ \text { Age } & 1.036 & 1.001-1.073 & 0.046 \\ \text { Aspirin use } & 1.552 & 0.642-3.750 & 0.329 \\ \text { Haemorrhagic stroke } & 8.076 & 2.223-29.346 & 0.002 \\ \text { NIHSS 8 or more } & 2.556 & 1.082-6.042 & 0.032\end{array}$

\section{Strength and Limitation}

Strength This is the first study concerning atherosclerotic ischaemic stroke patients with previous aspirin use with only a very small proportion of patients lost to follow up.

Limitation Small sample size; inadequate workup for large vessel disease due to limitation of public resources.

\section{Conclusion}

Prior aspirin use cannot be regarded as a surrogate risk factor for stroke recurrence and mortality. To compare the cardiovascular risk of patients with clinical aspirin failure to those without, a matched cohort study or can be planned in the future. 